\title{
Osteoactivin inhibition of osteoclastogenesis is mediated through CD44-ERK signaling
}

\author{
Gregory R Sondag ${ }^{1,2}$, Thomas S Mbimba ${ }^{1,2}$, Fouad M Moussa ${ }^{1,2}$, Kimberly Novak ${ }^{1,3}$, Bing $\mathrm{Yu}^{4}$, \\ Fatima A Jaber ${ }^{1,2,5}$, Samir M Abdelmagid ${ }^{1}$, Werner J Geldenhuys ${ }^{6}$ and Fayez F Safadi ${ }^{1,2,3,7}$
}

Osteoactivin is a heavily glycosylated protein shown to have a role in bone remodeling. Previous studies from our lab have shown that mutation in Osteoactivin enhances osteoclast differentiation but inhibits their function. To date, a classical receptor and a signaling pathway for Osteoactivin-mediated osteoclast inhibition has not yet been characterized. In this study, we examined the role of Osteoactivin treatment on osteoclastogenesis using bone marrow-derived osteoclast progenitor cells and identify a signaling pathway relating to Osteoactivin function. We reveal that recombinant Osteoactivin treatment inhibited osteoclast differentiation in a dose-dependent manner shown by qPCR, TRAP staining, activity and count. Using several approaches, we show that Osteoactivin binds CD44 in osteoclasts. Furthermore, recombinant Osteoactivin treatment inhibited ERK phosphorylation in a CD44-dependent manner. Finally, we examined the role of Osteoactivin on receptor activator of nuclear factor-K B ligand (RANKL)-induced osteolysis in vivo. Our data indicate that recombinant Osteoactivin inhibits RANKL-induced osteolysis in vivo and this effect is CD44-dependent. Overall, our data indicate that Osteoactivin is a negative regulator of osteoclastogenesis in vitro and in vivo and that this process is regulated through CD44 and ERK activation.

Experimental \& Molecular Medicine (2016) 48, e257; doi:10.1038/emm.2016.78; published online 2 September 2016

\section{INTRODUCTION}

As the aging population continues to increase, the prevalence of bone-related pathologies will also continue to increase. Osteoporosis is the most common cause of fractures with an estimated 33.6 million individuals over the age of 50 having osteopenia or low bone mass. ${ }^{1}$ Bone is a dynamic tissue consisting of a variety of cell types. Osteoblasts are the cells that secrete organic matrix and minerals that form bone. Osteoclasts are multinucleated cells that secrete degradative enzymes that result in the resorption of bone matrix. The processes of bone formation and bone resorption are collectively known as bone remodeling and occur throughout the aging process. ${ }^{2}$ This is a tightly coupled process that is regulated by a variety of molecules. In the case of osteoporosis, bone resorption becomes more active, whereas bone formation becomes less active. ${ }^{3}$ Currently, there are few therapeutic agents that help treat bone loss. The search for new therapeutics for the treatment of osteoporosis and bone loss has become an imperative aspect.
Through the use of genetically manipulated animal models to mimic disease state, new molecules important for bone development have been discovered. Osteoactivin (OA/GPNMB) was first discovered in a model of the op osteopetrotic rat. ${ }^{4}$ Osteoactivin is a heavily glycosylated type I transmembrane protein expressed in both osteoblasts and osteoclasts. ${ }^{5,6}$ Previous literature has shown that Osteoactivin undergoes ectodomain shedding by metalloproteinases and can stimulate different cellular functions. ${ }^{7-9}$ Our lab has shown that Osteoactivin is a positive regulator of bone formation in vitro and in vivo. ${ }^{5,10-12}$ Mutation in Osteoactivin resulted in impaired bone formation both in vivo and ex vivo. ${ }^{13}$ Osteoactivin has also shown to be expressed in osteoclasts. Our lab has shown that mutation in Osteoactivin results in enhanced osteoclastogenesis but impaired bone resorption indicating that Osteoactivin acts as a negative regulator of osteoclast differentiation, but a positive regulator of bone resorption. ${ }^{14}$ Other groups have shown that Osteoactivin acts as a positive regulator for both osteoclast differentiation and

\footnotetext{
${ }^{1}$ Department of Anatomy and Neurobiology, Northeast Ohio Medical University (NEOMED), College of Medicine, Rootstown, OH, USA; ${ }^{2}$ School of Biomedical Sciences, Kent State University, Kent, OH, USA; ${ }^{3}$ Department of Pharmaceutical Sciences, Northeast Ohio Medical University (NEOMED), College of Pharmacy, Rootstown, OH, USA; ${ }^{4}$ Department of Biological Sciences, Kent State University, Kent, OH, USA; ${ }^{5}$ Department of Biology, King Abdulaziz University, Jeddah, KSA; ${ }^{6}$ Department of Pharmaceutical Sciences, West Virginia University, Morgantown, WV, USA and ${ }^{7}$ Department of Orthopedics, Summa Health Systems, Akron, OH, USA

Correspondence: Professor FF Safadi, Department of Anatomy and Neurobiology, Northeast Ohio Medical University (NEOMED) College of Medicine, 4209 State Route 44, Rootstown, OH 44224, USA.

E-mail: fayez.safadi@neomed.edu

Received 22 December 2015; revised 24 March 2016; accepted 14 April 2016
} 
function. ${ }^{6,15,16}$ Thus, the role of Osteoactivin should be clearly defined in order for its potential use as a therapeutic option.

The signaling pathway involved in Osteoactivin-mediated regulation of osteoclast differentiation and function has yet to be completely and clearly defined. Previous studies have shown that Osteoactivin may bind to $\alpha_{v} \beta_{3}$ integrin and stimulate resorption. ${ }^{6,16}$ To date there has not yet been a receptor identified for Osteoactivin-mediated regulation of osteoclast differentiation. Recently, we have shown that Osteoactivin binds to CD44, a transmembrane receptor expressed in bone cells. ${ }^{9}$

The first evidence of CD44 expression in bone was shown in osteocytes and was proposed as a marker for osteocytogenesis. ${ }^{17}$ Since then, CD44 has been shown to be expressed in osteoblasts, ${ }^{18-20}$ mesenchymal stem cells ${ }^{21}$ and osteoclasts. $^{22}$ Studies from CD44-deficient mice show no difference in osteoclast differentiation both in vivo and in vitro; ${ }^{23-25}$ however, when challenged with TNF- $\alpha$ (tumor necrosis factor- $\alpha$ ), they develop inflammatory-induced bone loss. ${ }^{23} \mathrm{CD} 44$ has shown to have a large role in osteoclasts and osteoclast signaling. Several studies involving CD44 and osteoclasts have shown that CD44 interacts with $\alpha_{v} \beta_{3}$ and osteopontin. $^{26,27}$ Previous literature has shown that CD44 stimulation by hyaluronan inhibits the migration of osteoclasts by down regulating matrix matalloproteinases. ${ }^{28}$ These data indicate that CD44 activation may act as a 'stop' signal for osteoclast resorption. Furthermore, it has been shown that CD44 ligands, such as hyaluronan, chondroitin sulfate and osteopontin, prevent macrophage/monocyte multinucleation. ${ }^{29}$ This suggests that CD44 may be an important regulator of osteoclast size and fusion.

In this study, we used the recombinant Osteoactivin (rOA) in order to determine its role in osteoclastogenesis. We determined that recombinant Osteoactivin inhibits osteoclast differentiation of bone marrow-derived osteoclast progenitor cells in a dose-dependent manner in vitro assessed by TRAP staining-, activity-, and count as well as osteoclast-related gene expression. Furthermore, we reveal that recombinant Osteoactivin inhibits the ERK signaling pathway in osteoclasts through the CD44 receptor, and that Osteoactivin-mediated inhibition of osteoclastogenesis is CD44-dependent. Finally, we determined that Osteoactivin inhibits receptor activator of nuclear factor- $\kappa$ B ligand (RANKL)-induced osteolysis in vivo, and that this process is also CD44-dependent. Overall, we believe that Osteoactivin-regulated inhibition of osteoclast differentiation is mediated through the CD44-ERK signaling axis. This is the first study to examine the relationship between Osteoactivin and CD44 in osteoclasts.

\section{MATERIALS AND METHODS}

\section{Cell culture and reagents}

Recombinant Osteoactivin (rOA), RANKL, M-CSF and Hyaluronan were purchased from R\&D Technologies (Minneapolis, MN, USA). The CD44 antibody was purchased from Calbiochem (San Diego, CA, USA). The antibodies pPLC $\gamma 2$, PLC $\gamma 2, \mathrm{pI}_{K} \beta, \mathrm{I}_{\mathrm{K}} \beta, \mathrm{pJNK}$, JNK, pERK, ERK, pP38, P38 and GAPDH were purchased from Cell Signaling
(Danvers, MA, USA). The tubulin antibody was purchased from Bioss (Woburn, MA, USA). The p-nitrophenyl phosphate, Fast Red Violet Salt and WGA Lectin HRP (from Triticum vulgaris) were all purchased from Sigma (St Louis, MO, USA).

\section{Mice and osteoclast culture}

C57Blk6 (wild-type (WT)) and CD44-deficient mice (CD44-/-; $\mathrm{CD} 44 \mathrm{KO}$ ) were purchased from Jackson Laboratory (Bar Harbor, ME, USA). WT and CD44KO 8-week-old males were used for osteoclast culture. All mouse colonies were housed and maintained at Northeast Ohio Medical University according to the guidelines set by the Institutional Animal Care and Use Committee (IACUC). Mouse bone marrow cells were isolated from 8-week-old male WT and CD44KO mice as previously described. ${ }^{14}$ To generate osteoclasts, nonadherent bone marrow cells were plated at a cell density of $1.2 \times 10^{5}$ cells in a 96-well plate and primed with M-CSF $\left(20 \mathrm{ng} \mathrm{ml}^{-1}\right)$ for the first 3 days. Osteoclast precursor cells (OCPs) were then given RANKL (40 $\mathrm{ng} \mathrm{ml}^{-1}$ ) and M-CSF (20 $\mathrm{ng} \mathrm{ml}^{-1}$ ) and a second dose of RANKL and M-CSF was given again $48 \mathrm{~h}$ later. In parallel cultures, OCP were treated with recombinant Osteoactivin along with RANKL and M-CSF. In certain instances, experiments were terminated at specific time points that include Primed ( 1 dose of $20 \mathrm{ng} \mathrm{ml}^{-1} \mathrm{M}-\mathrm{CSF}$ ), Oc $5 \mathrm{~d}$ (second dose of M-CSF with firsst dose of RANKL $40 \mathrm{ng} \mathrm{ml}^{-1}$ ), and Oc 7d (third dose M-CSF with second dose of RANKL). At day 7 osteoclasts were fixed, and TRAP activity and staining was assessed. TRAP-positive osteoclast ( $>3$ nuclei) images were taken using a Nikon Ti Eclipse inverted microscope (Nikon Instruments Inc., Melville, NY, USA). TRAP-positive osteoclast differential count and size were calculated using the NIS-Elements software (Nikon Instruments Inc.).

\section{TRAP staining and activity}

Mature osteoclasts in 96-well plates were fixed with $10 \%$ formalin, washed with $\mathrm{dH}_{2} \mathrm{O}$ and allowed to air dry. For TRAP activity assays methanol:acetone was added to cultures followed by incubation with TRAP buffer ( $52 \mathrm{~mm}$ of Na-tartrate in $0.1 \mathrm{M} \mathrm{Na}$-acetate buffer $\mathrm{pH}$ 5.2) containing $0.1 \mathrm{mg} \mathrm{ml}^{-1}$ of $p$-nitrophenyl phosphate. The reaction was stopped by adding $1 \mathrm{~N} \mathrm{NaOH}$ and read at an optical density of $405 \mathrm{~nm}$ using a BioTek Synergy microplate reader (BioTek, Winooski, VT, USA). For TRAP staining, mature osteoclasts were incubated with TRAP buffer containing $1.5 \mathrm{~mm}$ naphthol AX-MX phosphate and $0.5 \mathrm{~mm}$ Fast Red Violet LB Salt.

\section{Immunoprecipitation}

Total cell lysates from osteoclast culture were pre-cleared with $20 \mu \mathrm{l}$ protein A-Sepharose beads (Sigma; 50\% slurry in phosphate-buffered saline (PBS)) end-over-end at $4{ }^{\circ} \mathrm{C}$ for $1 \mathrm{~h}$. Cell lysates were then incubated with $3 \mu \mathrm{g}$ anti-CD44 antibody or corresponding IgG isotype control (Abcam, Cambridge, UK) end-over-end at $4{ }^{\circ} \mathrm{C}$ overnight. The immune-complexes were captured by $25 \mu$ l protein A-Sepharose beads with end-over-end mixing for $1 \mathrm{~h}$ at $4{ }^{\circ} \mathrm{C}$. Beads were then washed four times by centrifugation $(400 \times g, 5 \mathrm{~min})$ in cell lysis buffer and then boiled at $95{ }^{\circ} \mathrm{C}$ for $5 \mathrm{~min}$ in $20 \mu \mathrm{l}$ reducing SDS-sample buffer inducing the elution of the captured proteins. Beads were removed by centrifugation, and the supernatant was analyzed by Western blot.

\section{Western blot analysis}

Total protein was isolated from osteoclast cultures using radioimmunoprecipitation assay buffer (Millipore, Billerica, MA, USA) with phosphatase inhibitor cocktail (Pierce, Rockford, IL, USA). Protein concentration was determined using the Pierce BCA protein 
Assay kit. Protein samples $(25-40 \mu \mathrm{g})$ were subjected to separation SDS-PAGE and transferred to polyvinylidene fluoride membranes (BioRad, Hercules, CA, USA) by the Trans-Blot Turbo system (BioRad). Membranes were probed overnight with primary antibodies against CD44, pPLC $\gamma 2$, PLC $\gamma 2, \mathrm{pI}_{\mathrm{K}} \beta, \mathrm{I}_{\mathrm{K}} \beta, \mathrm{pJNK}$, JNK, pERK, ERK, pP38, P38, GAPDH and Tubulin followed by incubation with secondary HRP conjugated antibodies. The signal was developed with chemiluminescent substrate (Millipore) and detected using the Syngene PXi system (Syngene, Rockville, MD, USA). Densitometric analysis was performed by the optical density of the phosphorylated protein over the total using the Syngene software analysis.

\section{Docking studies}

The docking studies used in this manuscript were conducted as previously described. ${ }^{30,31}$ Briefly, 3D-QSAR modeling was performed using the CoMFA modules of SYBYL 8.1 (Tripos, St Louis, MO, USA), running on a Dell XPS720 3.66 GHz PC (Dell, Round Rock, TX, USA), dual-booted to run Red Hat Linux Enterprise 5 (Red Hat, Raleigh, NC, USA), and Microsoft Windows XP (Microsoft, Redmond, WA, USA). Compounds were drawn in SYBYL, and exported as SDF files. The SDF files were then used by OMEGA 2.3.2 (OpenEye Scientific Software, Santa Fe, NM, USA, http://www.eyesopen.com) which generates multiple conformers of each compound. ROCS 2.4.1 (OpenEye Scientific Software, http://www.eyesopen.com; commandline mode) software from Openeye using the RANKBY COMBO flags which optimize both shape overlay and chemical (color) overlay. For the docking study, we used MOE-Dock (www.chemcomp.com). The protein was protonated at $\mathrm{pH} 7.4$, to assure biological relevance.

\section{In vitro Osteoclast resorption assays}

OCP isolated from WT and CD44KO were plated on Corning OsteoAssay surfaces (Corning, Corning, NY, USA) for 4 days with M-CSF $\left(20 \mathrm{ng} \mathrm{ml}^{-1}\right)$ and RANKL $\left(40 \mathrm{ng} \mathrm{ml}^{-1}\right)$. In parallel cultures, rOA ( $100 \mathrm{ng} \mathrm{ml}^{-1}$ ) was added to cultures $48 \mathrm{~h}$ before termination. On the fourth day, osteoclast cultures were terminated using $10 \%$ bleach. Resorption pits were analyzed by quantitation of the resorbed area of the well over the total area using the NIS-Elements software. In addition, OCP from WT and CD44KO were cultured on collagencoated six-well plates and treated with M-CSF $\left(20 \mathrm{ng} \mathrm{ml}^{-1}\right)$ and RANKL ( $40 \mathrm{ng} \mathrm{ml}^{-1}$ ) for $48 \mathrm{~h}$. Mature osteoclasts on collagen-coated plates were removed using $2.5 \mathrm{mg} \mathrm{ml}^{-1}$ collagenase in dissociation

Table 1 Osteoclast primers used for qPCR

\begin{tabular}{ll}
\hline Primers & \multicolumn{1}{c}{ Sequence } \\
\hline RANK & 5'-AGTTTAAGCCAGTGCTTCACG-3' (RANK forward) \\
& 5'-ACGTAGACCACGATG ATGTCG-3' (RANK reverse) \\
TRAP & 5'-GCAGTATCTTCAG GACGAGAAC-3' (TRAP forward) \\
& 5'-TCCATAGTGAAACCGCAAGTAG-3' (TRAP reverse) \\
DC- & 5'-TGTATCGGCTCA TCTCCTCCAT-3' (DC-STAMP forward) \\
STAMP & 5'-GACTCCTTGGGTTCCTTGCTT-3' (DC-STAMP reverse) \\
NFATc1 & 5'-CTCGAAAGACAGCAC TGGAGCAT-3' (NFATc1 forward) \\
& 5'-CGG CTGCCTTCCGTCTCATAG-3' (NFATc1 reverse) \\
OSCAR & 5'-CTGCTGGTAACGGATCAGTC CCCAGA-3' (OSCAR forward) \\
& 5'-CCAAGGAGCCAGAACCTTCGAAACT-3' (OSCAR reverse) \\
Calcitonin & 5'-AGTTGCCCTCTTATGAAGGAGAAG-3' (calcitonin receptor \\
receptor & forward) \\
& 5'-GGAGTGTCGTCCCAGCACAT-3' (calcitonin receptor \\
& reverse)
\end{tabular}

buffer (Life Technologies, Carlsbad, CA, USA) and seeded on bovine cortical slices in 96-well plates along with M-CSF and RANKL. In parallel cultures, rOA $\left(100 \mathrm{ng} \mathrm{ml}^{-1}\right)$ was added to osteoclasts seeded on cortical slices. Cells were fixed with $4 \%$ paraformaldehyde, permeabilized and incubated with rhodamine phalloidin, followed by counterstaining with DAPI mounting media to visualize actin ring formation. Images were taken using the Olympus 100 confocal microscope (Olympus, Tokyo, Japan). After staining for actin ring formation, osteoclasts on cortical slices were TRAP-stained and counted ( $>3$ nuclei) for each condition. For analysis of the resorption pits on the cortical slices, osteoclasts were removed with a soft brush and slices were incubated with $200 \mu \mathrm{g} \mathrm{ml}^{-1}$ lectin HRP for $1 \mathrm{~h}$ at room temperature. Slices were then incubated with $0.52 \mathrm{mg} \mathrm{ml}^{-1}$ $3,3^{\prime}$-diaminobenzidine for $30 \mathrm{~min}$. Images were taken using a Nikon Ti Eclipse inverted microscope and resorption pit area and \% of the resorbed area were measured using the NIS-elements software.

\section{Calvaria bone resorption assay}

A mouse model of RANKL-induced osteolysis from the calvaria was used as previously described. ${ }^{32}$ A collagen sheet $\left(100 \mathrm{~mm}^{2}\right.$; Medline, Mundelein, IL, USA) was soaked with PBS (control), RANKL $\left(50 \mu \mathrm{g} \mathrm{ml}^{-1}\right)$ or RANKL and rOA $\left(100 \mu \mathrm{g} \mathrm{ml}^{-1}\right)$ in $30 \mu \mathrm{l}$ volume and placed on the center of the calvaria of 5-week-old WT or CD44KO mice $(n=5)$. After 7 days, the mice were killed and the calvaria were removed and fixed in $4 \%$ paraformaldehyde and stained for TRAP. Images were taken using a Nikon SMZ 800 stereomicroscope (Nikon Instruments Inc.). The TRAP+stained area was quantified using the NIS-Elements analysis software. Microcomputated tomography was also performed using the SkyScan 1172 microCT system (Skyscan, Aartselaar, Belgium; $72 \mathrm{kV}, 113 \mu \mathrm{A}$, 7.7- $\mu \mathrm{m}$ sections). Three-dimensional reconstructed images of the calvaria were generated using SkyScan CTvox software (Skyscan).

\section{RT-qPCR}

Total RNA was isolated from OCP, pre-osteoclasts and mature osteoclasts grown in six-well dishes by adding $1 \mathrm{ml}$ Qiazol (QIAGEN, Hilden, Germany). Total RNA was purified using an RNA extraction kit (QIAGEN). RNA concentrations were determined using a NanoDrop 2000 Spectrophotometer (ThermoScientific, Pittsburgh, PA, USA). Following RNA isolation cDNA was prepared using a High Capacity cDNA Reverse Transcription kit (Life Technologies). Quantitative RT-PCR was performed with the Step-one qPCR system in duplicate with $2 \times$ SYBR Green PCR Master Mix (Life Technologies). qPCR cycles consisted of an initial $50^{\circ} \mathrm{C}$ cycle for $2 \mathrm{~min}$, followed by a second cycle of $95^{\circ} \mathrm{C}$ for $10 \mathrm{~min}$, followed by 40 cycles of $95^{\circ} \mathrm{C}$ for $15 \mathrm{~s}$ and a final cycle of $60^{\circ} \mathrm{C}$ for $1 \mathrm{~min}$. Samples were analyzed using a 7900 Fast Real Time PCR System (Applied Biosystems, Foster City, CA, USA), and relative mRNA expression of osteoclast-related genes was determined using the $\triangle \Delta \mathrm{C}_{\mathrm{T}}$ method using GAPDH as an internal control. The sequences for oligonucleotide primers (Integrated DNA technologies, Coralville, IA, USA) for osteoclast-related genes are listed in Table 1.

\section{RESULTS}

\section{Osteoactivin Inhibits RANKL-mediated Osteoclast Differentiation}

Previous studies have shown that Osteoactivin is expressed in osteoclasts. $^{6,14,15,33}$ The role of Osteoactivin in osteoclastogenesis has been somewhat controversial. Previous studies have shown that Osteoactivin is important for both osteoclast 
differentiation and function and that this process may be mediated through $\alpha_{\mathrm{v}} \beta_{3}$ integrin. ${ }^{14-16}$ Recently, our lab has found that mutation in Osteoactivin in D2J mice results in enhanced osteoclast differentiation. ${ }^{6}$ This indicates that Osteoactivin modulates osteoclastogenesis. Although there are a few reports regarding the role of Osteoactivin in osteoclasts, the downstream signaling pathways related to Osteoactivin have not yet been determined.

In order to differentiate osteoclasts in vitro, inflammatory cytokines M-CSF and RANKL are required. ${ }^{34}$ Certain cytokines have different effects on osteoclast differentiation based on when they are added in culture. ${ }^{35,36}$ In order to determine the role of exogenous Osteoactivin treatment on osteoclast differentiation, we added recombinant Osteoactivin to OCP during osteoclast differentiation. Previous work from our lab has shown that recombinant Osteoactivin has different effects on osteoclast differentiation depending on when it is added in culture (Sondag, 2014, unpubl data). In this study, recombinant Osteoactivin was added at day 3 and day 5 of osteoclast differentiation just prior to and during osteoclast commitment, respectively (Figure 1a). Exogenous Osteoactivin treatment inhibited osteoclast differentiation assessed by TRAP staining (Figure 1b) and activity (Figure 1c) in a dose-dependent manner. Furthermore, recombinant Osteoactivin significantly inhibited osteoclast size and number (Figures 1d-g). Next, we sought to determine the effect of recombinant Osteoactivin treatment on osteoclast-related gene expression. Osteoactivin treated OCP showed a significant reduction in osteoclastrelated gene expression both in a dose-dependent (Figure 1h) and time-dependent manner (Figure 1i). In order to determine at which stage of osteoclast differentiation Osteoactivin exhibits its maximum effect, recombinant Osteoactivin was added to osteoclasts in a stage-specific manner. Our results show that recombinant Osteoactivin treatment significantly inhibits osteoclast differentiation markers RANK and NFATc1, but slightly enhances osteoclast resorption marker Cathepsin $\mathrm{K}$ (Figures $1 \mathrm{j}$ and $\mathrm{k}$ ). These data suggest that recombinant Osteoactivin inhibits RANKL-mediated osteoclast differentiation in vitro in a stage-dependent manner.

\section{Recombinant osteoactivin inhibits ERK signaling in RANKL-induced osteoclastogenesis}

The importance of the RANKL pathway including downstream signaling mediators and transcription factors has been previously described. ${ }^{34,37,38}$ In order to determine the role of Osteoactivin during RANKL-mediated signaling, we pretreated osteoclasts with recombinant Osteoactivin followed by RANKL treatment at different time points and analyzed downstream signaling. Recombinant Osteoactivin treatment significantly reduced the levels of phosphorylated ERK compared with untreated controls (Figure 2a). Interestingly, Osteoactivin seemed to have no effect on phosphoJNK (Figure 2b), phosphoP38 (Figure 2c) or phosphoI ${ }_{K} \beta$ (Figure 2e) signaling. Interestingly, Osteoactivin treatment slightly increased the level of phosphoPLC 2 (Figure 2d); however, the increase was not significant due to protein loading. These results indicate that Osteoactivin specifically inhibits RANKL-mediated ERK activation and osteoclast differentiation.

\section{Osteoactivin-mediated inhibition of osteoclastogenesis is abrogated in CD44-deficient osteoclasts}

In order to further examine the role of Osteoactivin-CD44 interaction on osteoclastogenesis, we used WT and CD44deficient mice for osteoclast culture. We confirmed absence of CD44 in cultures by qPCR (Figure 3a) and Western blot (Figure 3b) analyses. Recombinant Osteoactivin added to WT cells showed a significant reduction in osteoclast differentiation by TRAP staining, activity and count in a dose-dependent manner; however, this effect was abrogated when CD44-deficient osteoclasts were treated with recombinant Osteoactivin (Figures $3 \mathrm{c}-\mathrm{e}$ ). This indicates that CD44 is involved in the Osteoactivin-mediated inhibition of osteoclastogenesis. To elaborate upon this idea, we repeated this same experiment with osteoclasts grown on bovine cortical slices. The results showed similar responses to osteoclasts grown on plastic. Osteoactivin inhibited osteoclast differentiation in WT cells as assessed by TRAP staining, activity and count; however, this effect was abrogated in CD44-deficient cells (Figures $3 \mathrm{f}-\mathrm{h}$ ). This indicates that osteoclasts from WT and CD44KO treated with Osteoactivin show similar results in both plastic and bovine slices. Next, we assessed whether CD44 was responsible for the Osteoactivin-mediated inhibition of osteoclast gene expression. Recombinant Osteoactivin added to WT OCP inhibited osteoclast-related genes including TRAP (Figure 3i), NFATc1 (Figure 3j), RANK (Figure 3k), DC-STAMP (Figure 31) and Calcitonin Receptor (Calcitonin Rec.; Figure $3 \mathrm{~m}$ ); however, when Osteoactivin was added to CD44-deficient osteoclasts, this effect was abrogated. These data suggest that recombinant Osteoactivin-mediated inhibition of osteoclast-related genes is CD44-dependent.

\section{Osteoactivin and hyaluronan inhibition of RANKL-induced osteoclastogenesis is mediated through two independent pathways}

Previous work from our lab has shown that Osteoactivin binds to and interacts with CD44 in mesenchymal stem cells. ${ }^{9}$ In order to gain insight into the interaction between CD44 and Osteoactivin, we conducted docking studies using the MOE-Dock program (www.chemcomp.com). ${ }^{30}$ The protein was protonated at $\mathrm{pH} 7.4$ to assure biological relevance. As can be seen in Figure 4a, the extracellular domain of Osteoactivin (OA-ECD; gray) interacts with the extracellular domain of CD44 (red). Interestingly, the specific interaction between OA-ECD and CD44 (green) occurs at the CD44 hyaluronanbinding domain. ${ }^{39}$ To further elucidate on the CD44Osteoactivin interaction, we used competitive binding studies using high-molecular-weight hyaluronan. Hyaluronan is the well-known and major ligand of CD44. ${ }^{40}$ In order to assess Osteoactivin affinity for CD44, OCP were treated with M-CSF and RANKL to generate mature osteoclasts. Osteoclasts were either left untreated or treated with biotinylated recombinant 
a

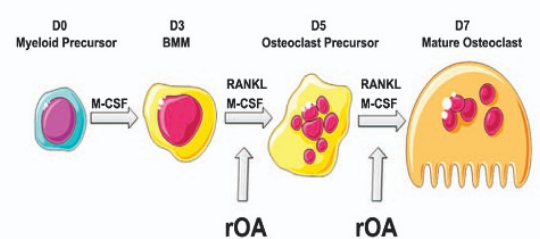

b

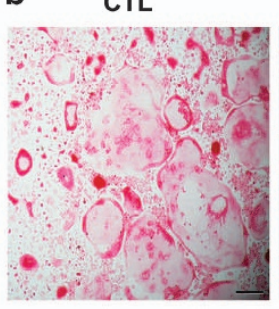

rOA $10 \mathrm{ng} / \mathrm{mL}$

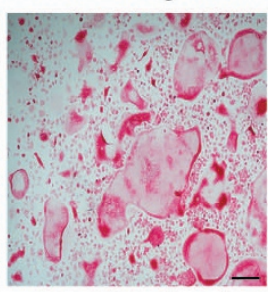

rOA 50ng/mL

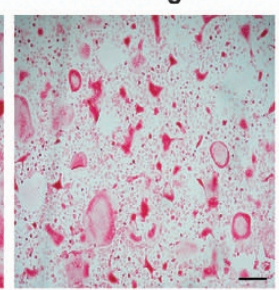

rOA $100 \mathrm{ng} / \mathrm{mL}$

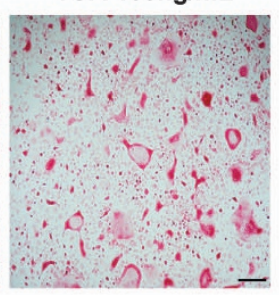

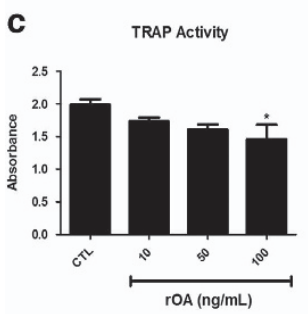
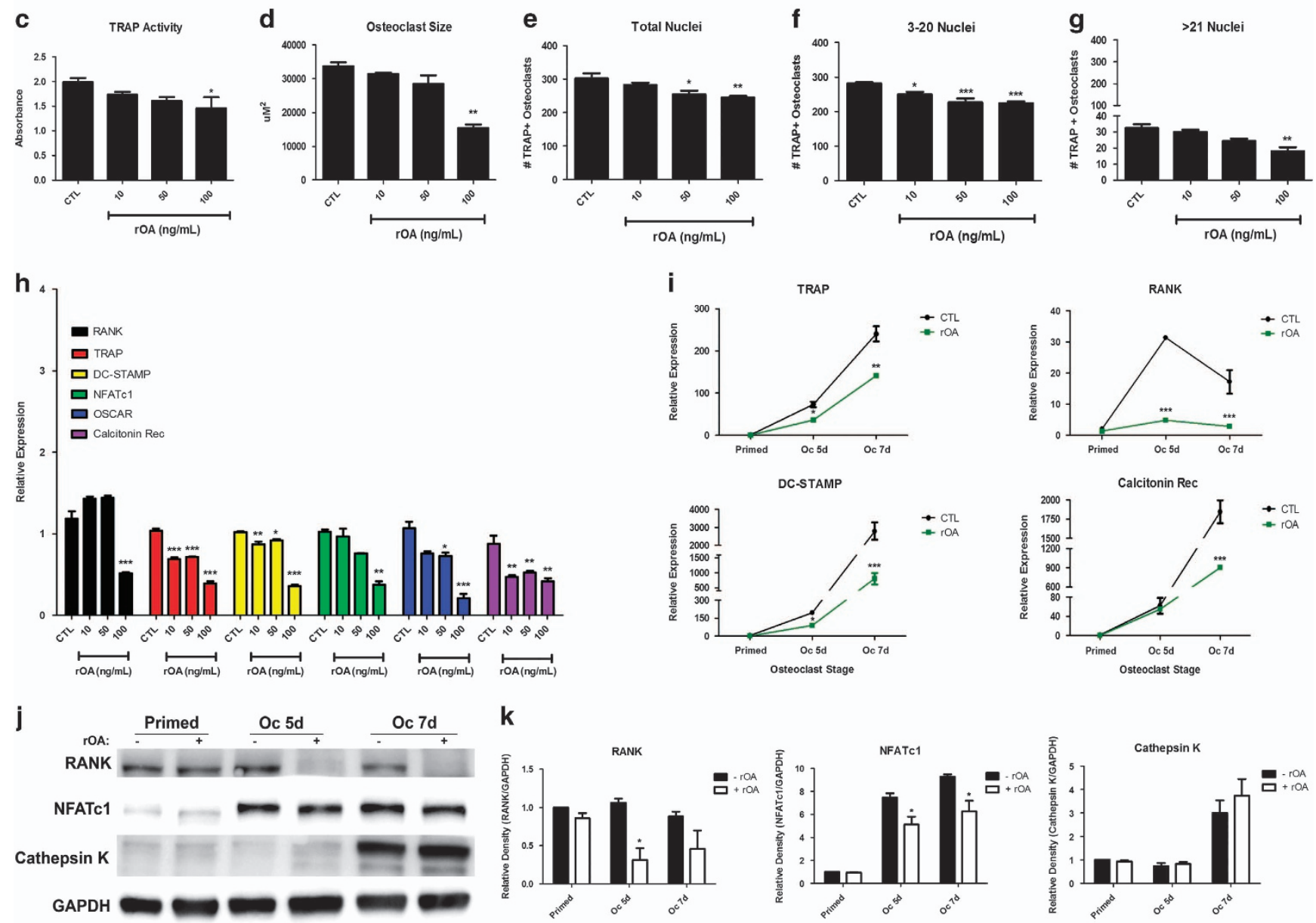

Figure 1 Recombinant Osteoactivin inhibits receptor activator of nuclear factor-א B ligand (RANKL)-mediated osteoclast differentiation in vitro. (a) Diagram depicting the treatment of M-CSF, RANKL and recombinant Osteoactivin (rOA) during osteoclast culture. C57BIk6 bone marrow-derived osteoclast precursor cells (OCPs) were cultured with M-CSF (20 ng ml-1) and RANKL (40 ng ml-1) with or without different doses of recombinant Osteoactivin and stained for TRAP (b). Osteoclast differentiation was assessed by TRAP activity (c), Osteoclast size (d), total count (total Nuclei; $n>3$ ) (e) and differential count (f, g). (h, i) Quantitative RT-PCR (qPCR) analysis of osteoclast-related genes is inhibited by recombinant Osteoactivin treatment in a dose-dependent (h) and a time-dependent (i) manner. (j, k) Western blot (j) and relative density analysis (k) of Osteoactivin treatment during different stages of osteoclastogenesis. Osteoactivin treatment inhibits osteoclast differentiation (RANK and NFATc1), but stimulates resorption (Cathepsin K) markers. These data are representative of three independent experiments. Data presented in all graphs represent mean \pm s.e.m. ${ }^{*} P<0.05$; ${ }^{* *} P<0.01$; $* * * P<0.001$ compared with control. Scale bar: $250 \mu \mathrm{m}$ (b).

Osteoactivin alone or in combination with high-molecularweight hyaluronan. Protein cell lysate was collected followed by immunoprecipitation. Immunoprecipitation analysis revealed that biotinylated recombinant Osteoactivin binds to CD44 and upon hyaluronan treatment, Osteoactivin and CD44 binding is reduced (Figure 4b). This indicates that Osteoactivin and hyaluronan may bind to $\mathrm{CD} 44$ at the same site.
Previous literature has shown that high-molecular-weight hyaluronan inhibits osteoclast differentiation through toll-like receptor 4 in vitro. ${ }^{32}$ In order to determine the interaction between hyaluronan, CD44 and Osteoactivin during osteoclastogenesis, we added either recombinant Osteoactivin or hyaluronan alone or in combination during osteoclast differentiation to WT and CD44-deficient mice (CD44KO). 


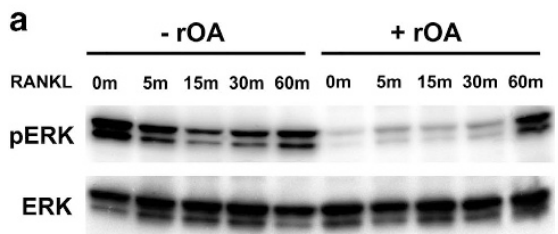

ERK

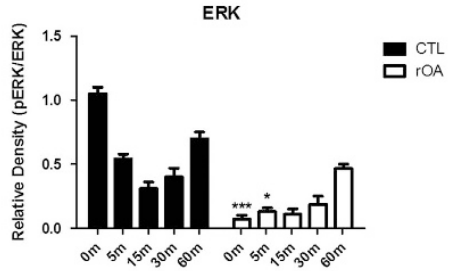

b

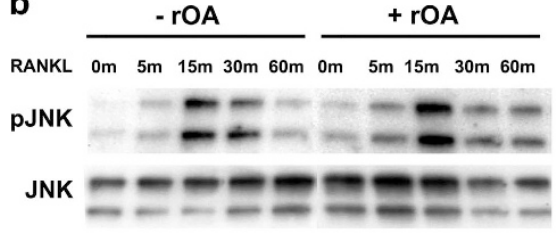

JNK

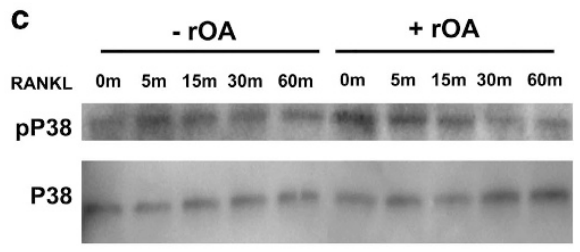

P38
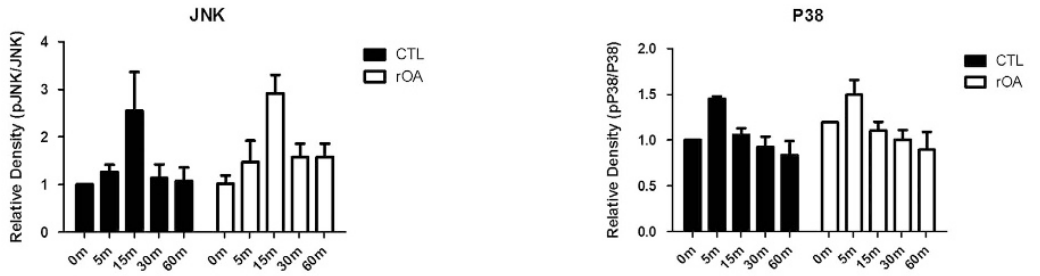

d

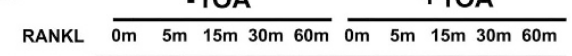
pPLCY2 - - - - - - - - PLCY2

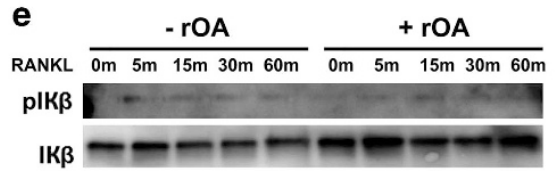

$\operatorname{l\kappa } \beta$

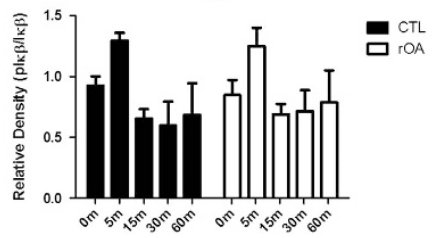

Figure 2 Recombinant Osteoactivin inhibits receptor activator of nuclear factor- $\mathrm{B}$ ligand (RANKL)-mediated osteoclast differentiation through the ERK pathway. (a) C57Blk6 bone marrow-derived osteoclast precursor cells (OCPs) were cultured with $\mathrm{M}^{-C S F}\left(20 \mathrm{ng} \mathrm{ml}^{-1}\right.$ ), RANKL (40 $\mathrm{ng} \mathrm{ml}^{-1}$ ) alone or with recombinant Osteoactivin (100 $\mathrm{ng} \mathrm{ml}{ }^{-1}$ ) for $48 \mathrm{~h}$. Recombinant Osteoactivin significantly inhibited ERK activation (a); however, other signaling molecules including JNK, P38, PLC $\gamma 2$ and I $\mathrm{\beta}$ did not seem to be affected (b-e). These data are representative of three independent experiments. Data presented in all graphs represent mean \pm s.e.m. ${ }^{*} P<0.05 ;{ }^{* *} P<0.001$ compared with control.

Recombinant Osteoactivin or hyaluronan treatment alone inhibited WT osteoclasts as expected. Interestingly, addition of Osteoactivin to CD44-deficient cells had no effect on osteoclast differentiation compared with control as assessed by TRAP staining and count (Figures $4 \mathrm{c}$ and d). Hyaluronan treatment to CD44-deficient cells alone inhibited osteoclasts, which was further enhanced with recombinant Osteoactivin. This indicates that Osteoactivin may inhibit osteoclasts through CD44 and that hyaluronan inhibits osteoclasts through toll-like receptor 4 and not CD44 as previously described.

\section{Osteoactivin inhibition of RANKL-induced ERK phosphorylation is mediated through CD44}

In order to determine if CD44 is responsible for Osteoactivinmediated ERK inhibition we used WT and CD44-deficient mice and examined RANKL-mediated stimulation of osteoclast signaling in response to Osteoactivin treatment. Previous literature has shown that absence of CD44 has no effect on osteoclast differentiation in vitro. ${ }^{25}$ Similarly, we have shown that absence of CD44 has no effect on RANKL-mediated ERK signaling (Figure 5a). In this study, we have shown that addition of recombinant Osteoactivin in RANKL-stimulated osteoclasts inhibits ERK phosphorylation. Addition of Osteoactivin in WT cells inhibited ERK phosphorylation as expected. Interestingly, the addition of Osteoactivin to CD44-deficient osteoclasts resulted in restoration of ERK phosphorylation to control levels (Figure 5b). This indicates that Osteoactivin inhibition of RANKL-induced ERK phosphorylation is mediated through CD44.

Recombinant osteoactivin inhibits osteoclast differentiation and recruitment through CD44 in vivo

Previous studies have shown that addition of antiinflammatory cytokines can inhibit osteoclast differentiation and migration using a calvaria osteolysis model. ${ }^{41-44}$ Therefore, we sought to examine the effects of recombinant Osteoactivin on osteoclast differentiation and recruitment in vivo. In WT mice, addition of RANKL significantly increased the number of TRAP-positive osteoclasts as expected; however, addition of RANKL in combination with recombinant Osteoactivin abrogated the RANKL-induced osteoclast differentiation and recruitment determined by TRAP staining (Figures $6 \mathrm{a}$ and b) and micro-computated tomography analysis (Figure 6c). To further elucidate the mechanism involved in the Osteoactivinmediated inhibition of osteoclastogenesis in vivo, we tested recombinant Osteoactivin using the same model in CD44deficient mice. As expected, RANKL alone stimulated osteoclast differentiation and recruitment. Interestingly, RANKL 


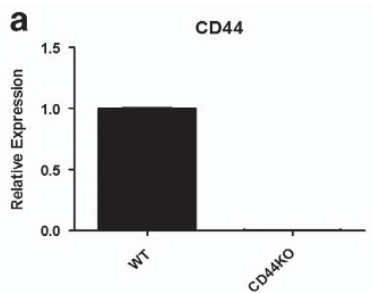

C

WT

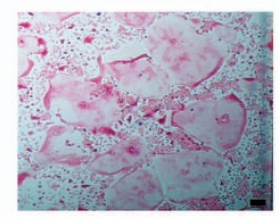

CD44KO

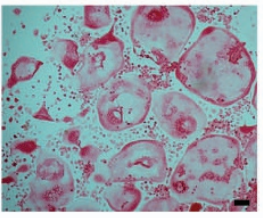

f
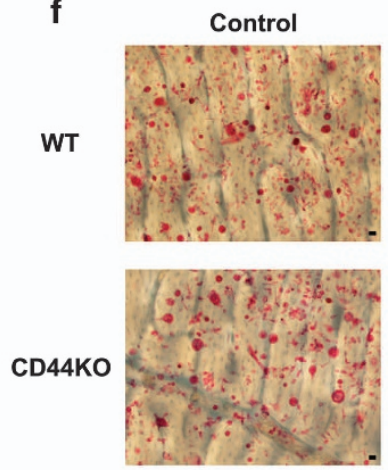

i
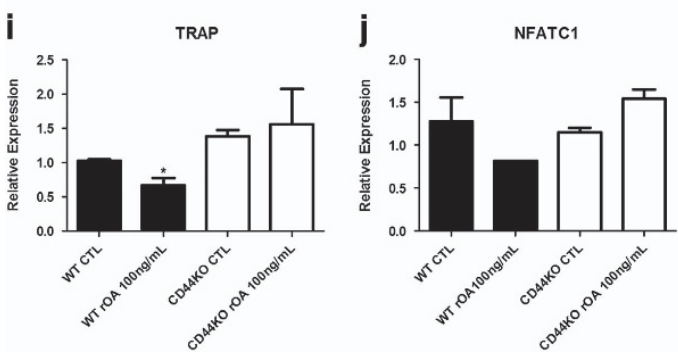

rOA $100 \mathrm{ng} / \mathrm{mL}$
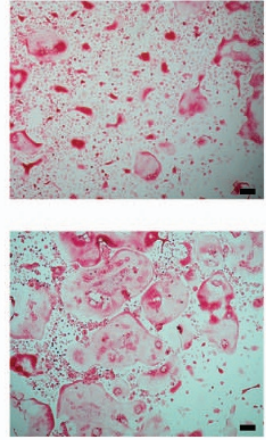

rOA $100 \mathrm{ng} / \mathrm{mL}$
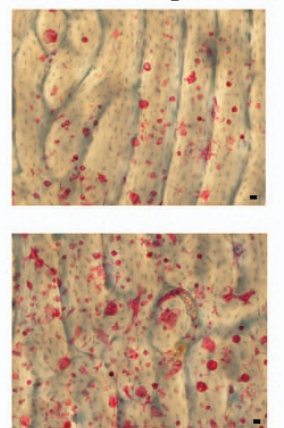

g

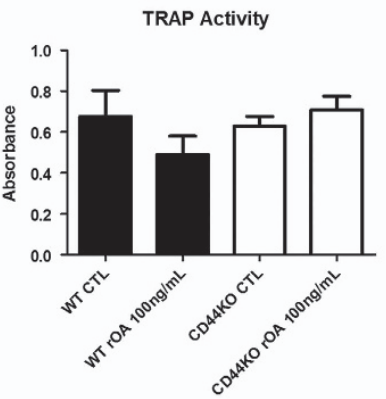

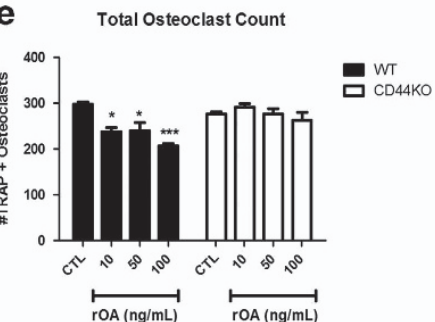

h

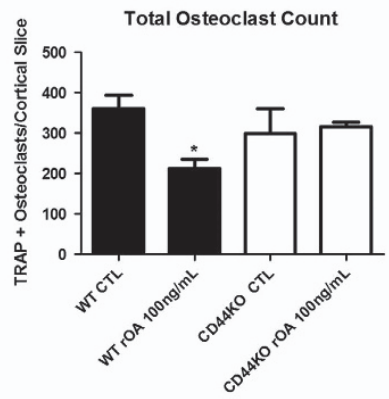

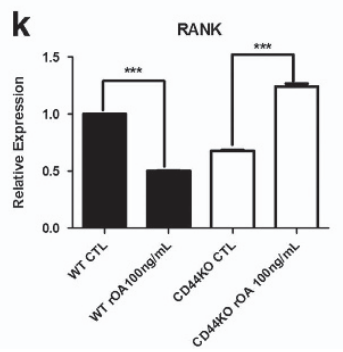
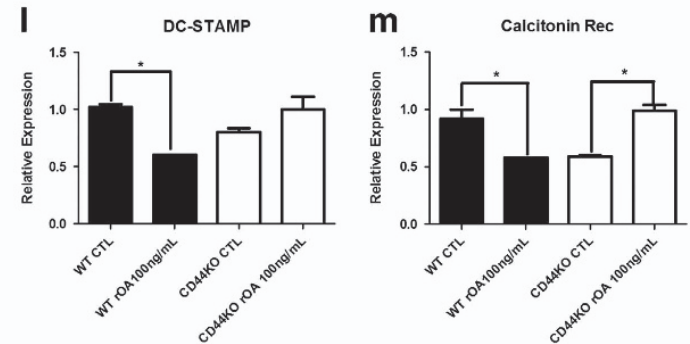

Figure 3 Osteoactivin inhibition of receptor activator of nuclear factor- $\kappa$ B ligand (RANKL)-mediated osteoclastogenesis is CD44dependent. (a, b) Confirmation of CD44 deficiency in CD44KO cells determined by quantitative RT-PCR (qPCR) (a) and western blot analysis (b). (c-e) Bone marrow-derived osteoclast precursor cells (OCPs) from wild-type (WT) and CD44KO cells were differentiated with M-CSF and RANKL alone (control) or in combination with recombinant Osteoactivin ( $\mathrm{rOA} ; 100 \mathrm{ng} \mathrm{ml}^{-1}$ ) for 7 days to generate mature osteoclasts. (c) Microscopic images of TRAP-positive osteoclasts show an inhibition upon rOA treatment in WT samples; however, in CD44deficient osteoclasts there is no effect upon rOA treatment. (d, e) Osteoclast parameters reveal an inhibition of osteoclast differentiation in rOA-treated WT cells shown by TRAP activity $(\mathbf{d})$ and osteoclast count $(n<3)(\mathbf{e})$. ( $\mathbf{f}-\mathbf{h})$ OCP from WT and CD44KO cells were cultured on collagen-coated six-well plates treated with M-CSF and RANKL for $48 \mathrm{~h}$ and re-plated onto bovine cortical slices with M-CSF and RANKL alone or with recombinant Osteoactivin for an additional $48 \mathrm{~h}$. Mature osteoclasts from WT and CD44KO were analyzed by TRAP staining (f) and activity (g) and total count $(n<3)(\mathbf{h})$. (i-m) qPCR analysis of osteoclast-related genes from WT and CD44KO osteoclasts treated with and without recombinant Osteoactivin. OCP from WT and CD44KO cells were cultured with M-CSF and RANKL alone (CTL) or with recombinant Osteoactivin for $48 \mathrm{~h}$ followed by RNA isolation and qPCR analysis for osteoclast-related genes TRAP (i), NFATc1 (j), RANK $(\mathbf{k})$, DC-STAMP $(\mathbf{I})$ and calcitonin receptor $(\mathbf{m})$. These data are representative of three independent experiments. Data presented in all graphs represent mean \pm s.e.m. ${ }^{*} P<0.05 ;{ }^{* * *} P<0.001$ compared with control. Scale bar: $100 \mu \mathrm{m}$ (c); $50 \mu \mathrm{m}$ (f). 
a

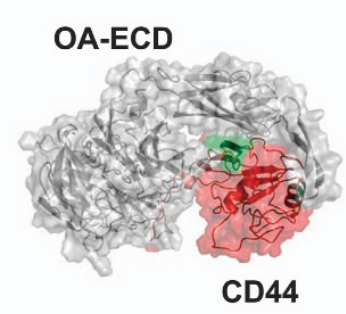

b

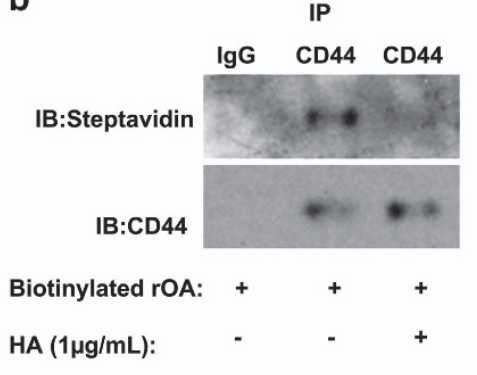

C

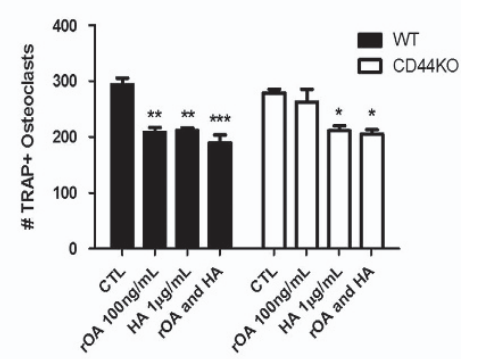

d

CTL

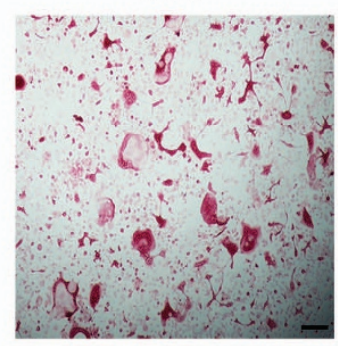

rOA $100 \mathrm{ng} / \mathrm{mL}$

HA $1 \mu \mathrm{g} / \mathrm{mL}$
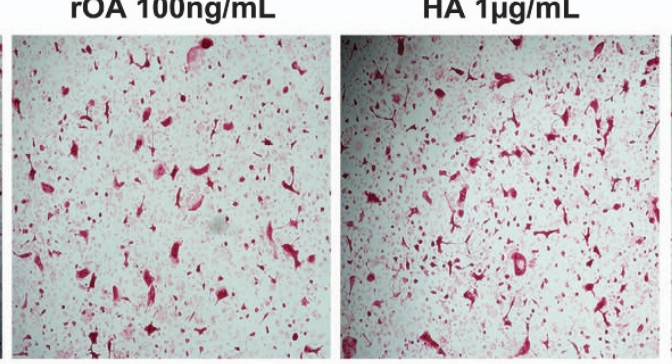

rOA + HA
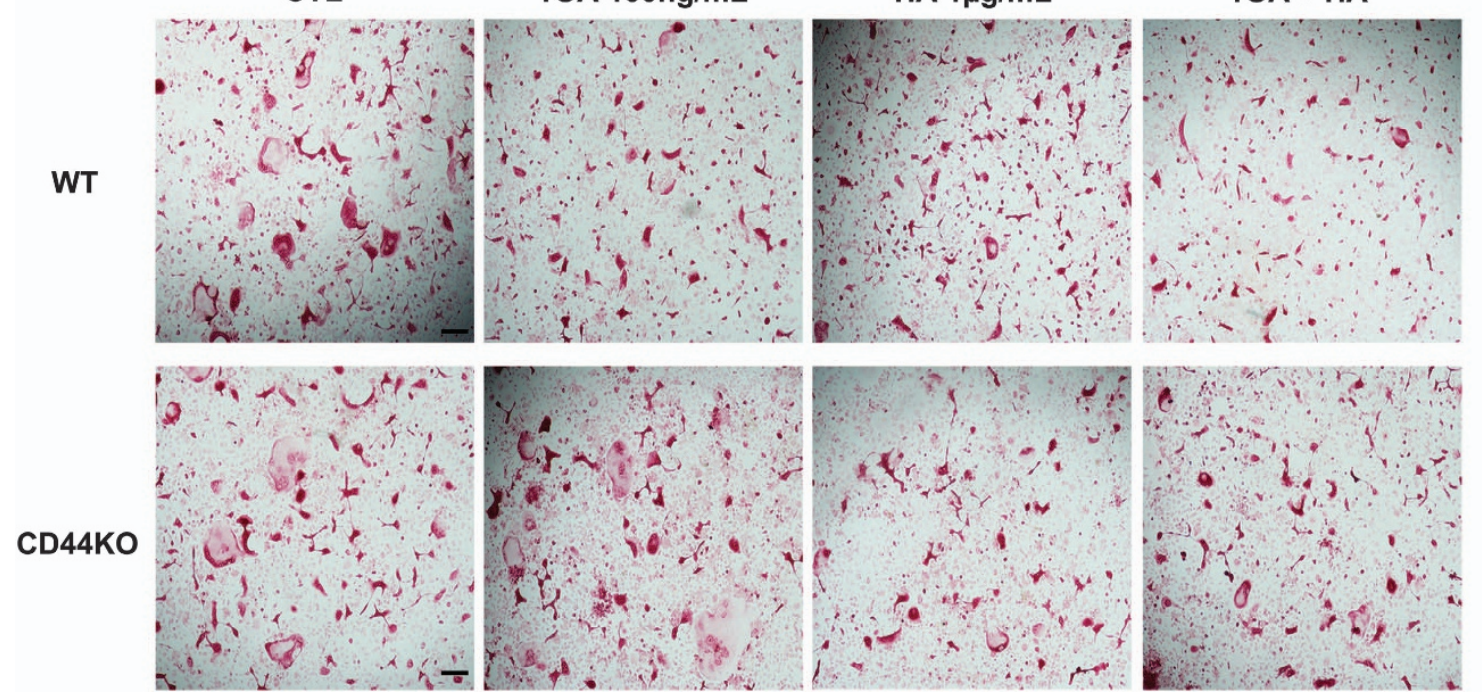

Figure 4 Osteoactivin and hyaluronan inhibit osteoclastogenesis through independent pathways. (a) Docking results from MOE suggesting interaction between Osteoactivin extracellular domain (OA-ECD; gray) and CD44 (red) at the CD44 hyaluronic acid-binding domain (green). (b) Immunoprecipitation analysis revealing the interaction between biotinylated recombinant Osteoactivin (rOA) and CD44. Osteoclast precursor cells (OCPS) from wild-type (WT) cells were differentiated into pre-osteoclasts with M-CSF and receptor activator of nuclear factor- $\kappa$ B ligand (RANKL) for $48 \mathrm{~h}$. After the $48 \mathrm{~h}$ period, osteoclasts were serum-starved and treated with biotinylated rOA alone or in combination with hyaluronan (HA). Protein cell lysates were collected and subjected to immunoprecipitation and Western blot analysis. Blots were probed with anti-streptavidin and anti-CD44. (c, d) OCP from WT and CD44KO cells were differentiated with M-CSF and RANKL alone (CTL) or with rOA (100 $\mathrm{ng} \mathrm{ml}^{-1}$ ) and hyaluronan ( $\mathrm{HA} ; 1 \mu \mathrm{g} \mathrm{ml} \mathrm{l}^{-1}$ ) alone, or in combination ( $\left.\mathrm{rOA}+\mathrm{HA}\right)$ for 7 days to generate mature osteoclasts. Osteoclast differentiation was assessed by TRAP count (c) and staining (d). These data are representative of three independent experiments. Data presented in all graphs represent mean \pm s.e.m. ${ }^{*} P<0.05,{ }^{* *} P<0.01,{ }^{* *} P<0.001$ compared with control. Scale bars: $250 \mu \mathrm{m}(\mathbf{d})$.

treatment, in combination with Osteoactivin, also stimulated osteoclast differentiation and recruitment in the CD44deficient mice. These data indicate that Osteoactivin inhibits RANKL-induced osteoclastogenesis through CD44-mediated signaling in vivo.

\section{DISCUSSION}

In this study, we showed that recombinant Osteoactivin inhibits RANKL-mediated osteoclast differentiation, size and osteoclast-related gene expression. Our lab was the first to show that mutation in Osteoactivin results in enhanced osteoclastogenesis. $^{14}$ Controversially, other groups have published that Osteoactivin stimulates both osteoclast differentiation and function., ${ }^{6,15,16}$ This may be explained by the fact that Osteoactivin may regulate osteoclasts in a stagespecific manner. Results generated from our lab have shown that addition of recombinant Osteoactivin has different effects on osteoclast differentiation and function based on when the protein is added in culture. Our results confirm the fact that Osteoactivin is a negative regulator of osteoclast differentiation through inhibition of RANK and NFATcl during early osteoclastogenesis, and moderately stimulates osteoclast resorption during osteoclast maturity by enhancing Cathepsin $\mathrm{K}$ expression.

In this study, we demonstrated that recombinant Osteoactivin treatment inhibits osteoclast differentiation in vitro and in vivo through a CD44-ERK-dependent mechanism. Previous data from our lab have shown that mutation in Osteoactivin results in enhanced osteoclast differentiation and survival. ${ }^{14}$ The MAPK pathway has extensively been shown to be important for osteoclast differentiation. ${ }^{45-48}$ Our data indicate that recombinant 
a

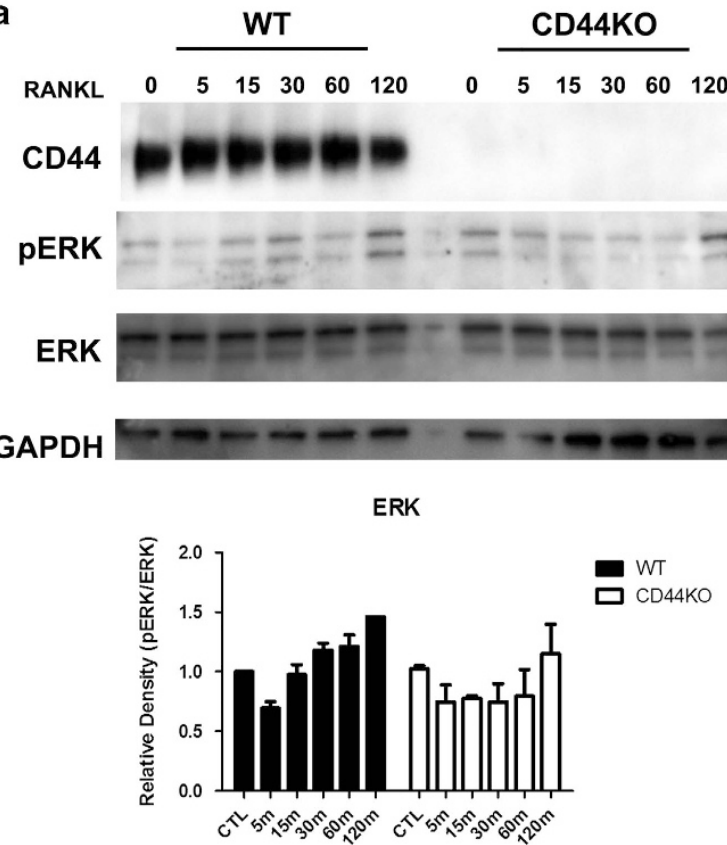

b

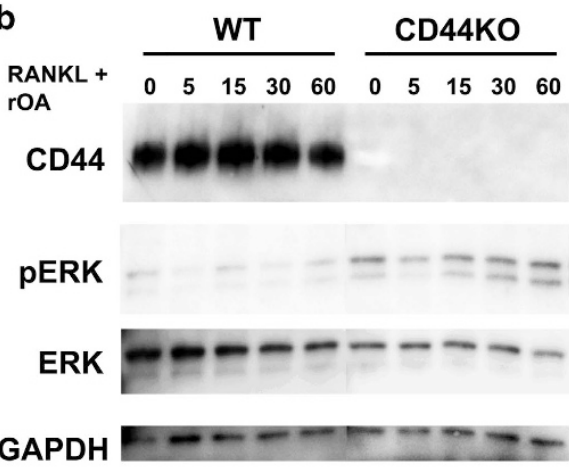

ERK

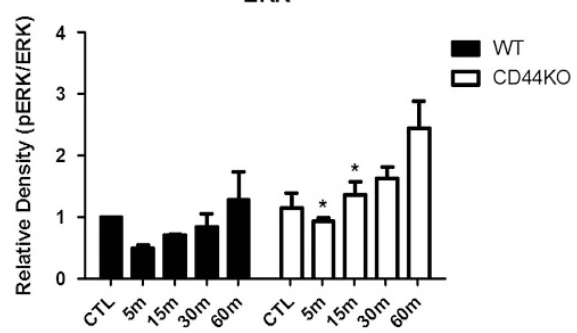

Figure 5 Osteoactivin-mediated inhibition of ERK signaling in osteoclasts is mediated through CD44. (a) Osteoclast precursor cells (OCPs) from wild-type (WT) and CD44KO mice were isolated and differentiated into osteoclasts with M-CSF and receptor activator of nuclear factor- $\mathrm{B}$ ligand (RANKL) for $48 \mathrm{~h}$. Protein cell lysates from the indicated time points were isolated, analyzed by western blot and probed with anti-CD44, anti-pERK, anti-ERK and anti-GAPDH. The results were analyzed using densitometric analysis of both the phosphoERK and total ERK bands. (b) Similarly, OCP from WT and CD44KO mice were isolated and differentiated into osteoclasts with M-CSF, RANKL and recombinant Osteoactivin for $48 \mathrm{~h}$. Protein cell lysates were isolated were analyzed by Western blot. Relative densitometry shows a restoration in the levels of pERK in CD44KO lysates. The data are representative of three independent experiments. Data presented in all graphs represent mean \pm s.e.m. ${ }^{*} P<0.05$ compared with control.

Osteoactivin inhibits RANKL-induced ERK activation in osteoclasts. Previous literature has shown that TNF- $\alpha$ and IL- $1 \alpha$ stimulate osteoclast survival through ERK signaling. ${ }^{49,50}$ It will be interesting to determine if recombinant Osteoactivin has any role in MEK signaling and if recombinant Osteoactivin regulates osteoclast survival through the MEK/ERK pathways in osteoclasts.

In the current study, we have shown that Osteoactivin interacts with CD44 in osteoclasts. Previous studies have shown that absence of CD44 has no effect on osteoclastogenesis; ${ }^{25}$ however, when challenged with an inflammatory cytokine such as TNF- $\alpha$, develop inflammatory bone loss. ${ }^{23}$ Furthermore, engagement of CD44 with hyaluronan was found to inhibit matrix matalloproteinase- 9 mRNA expression and result in the inhibition of osteoclast motility. ${ }^{28}$ Similarly, our results show that Osteoactivin interacts with CD44 in osteoclasts and that Osteoactivin-mediated inhibition of osteoclasts is CD44dependent. CD44 has also been shown to be an important fusion marker, ${ }^{51}$ and upon engagement with another ligand; inhibits multinucleation. ${ }^{29}$ Similarly, our data show that recombinant Osteoactivin inhibits osteoclast size which seems to be CD44-dependent. CD44 has been shown to have a role in osteoclast migration and adhesion. ${ }^{52}$ It will be interesting to determine if Osteoactivin has a role in osteoclast motility.

CD44 has shown to have a role in osteoclast-mediated bone resorption. Several sources of literature have shown that CD44 along with $\alpha_{\mathrm{v}} \beta_{3}$ integrin regulate osteoclast motility and bone resorption. ${ }^{26,53,54}$ Osteoactivin has shown to bind to $\alpha_{\mathrm{v}} \beta_{3}$ and is believed to be responsible for osteoclast-mediated resorption. ${ }^{6,16}$ In our study, we show that Osteoactivin binds to CD44; however, our data suggests that CD44 does not have a role in osteoclast-mediated resorption in vitro (Supplementary Figure 1). This may be explained by the fact that CD44 and $\alpha_{v} \beta_{3}$ integrin organize two functionally distinct domains in osteoclasts. ${ }^{55}$ In a previous study, CD44 was shown to occupy the podosome core, whereas $\alpha_{\mathrm{v}} \beta_{3}$ integrin occupies the outer F-actin cloud in osteoclasts. Therefore, it may be possible that Osteoactivin binds to CD44 and inhibits osteoclast differentiation and fusion, but also binds to $\alpha_{\mathrm{v}} \beta_{3}$ integrin and stimulates osteoclast resorption in vitro. Future studies should focus on particular interactions and binding specificities between Osteoactivin, CD44 and $\alpha_{\mathrm{v}} \beta_{3}$ in modulating osteoclast function.

Our data suggest that recombinant Osteoactivin inhibits osteoclast differentiation, but stimulates resorption in vitro. Several other extracellular matrix proteins have shown to have this similar effect on osteoclasts. Fibrillin-1 has been shown to have dual roles in regulating osteoclast differentiation and function. ${ }^{56}$ In this study, recombinant N-terminal Fibrillin-1 was shown to inhibit osteoclast differentiation but stimulate its function in vitro. Previous literature has also shown that fibronectin inhibits osteoclast differentiation activity, 
a
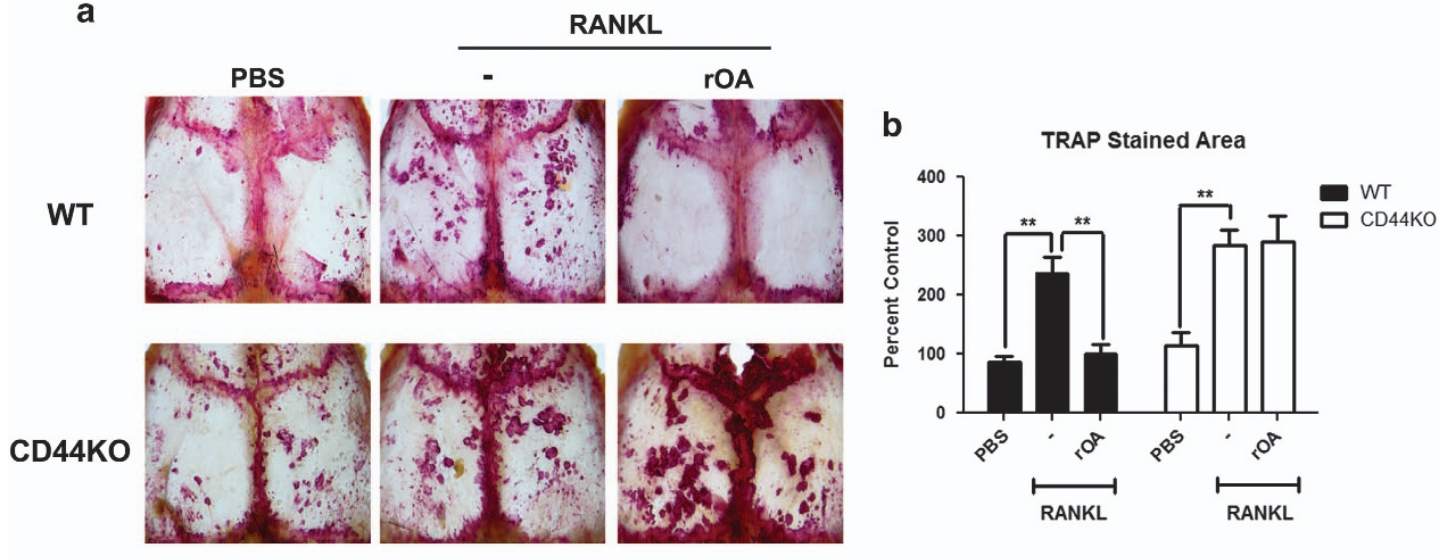

C

RANKL

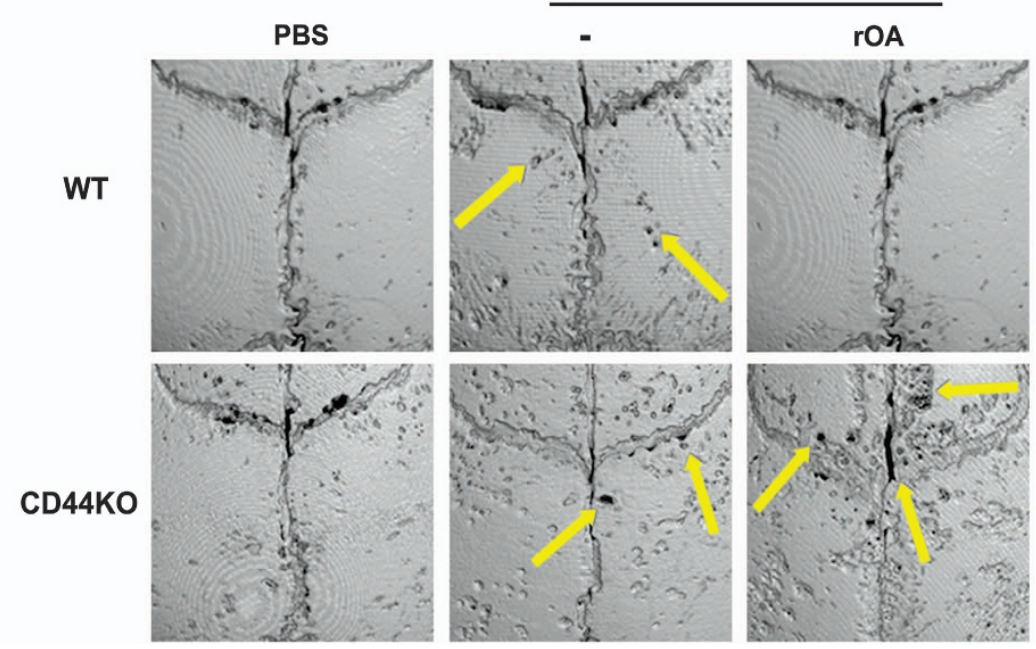

Figure 6 Recombinant Osteoactivin inhibition of receptor activator of nuclear factor-к B ligand (RANKL)-mediated osteoclast differentiation and recruitment in vivo is CD44-dependent. (a) Mouse calvarias $(n=5)$ were implanted with a collagen sheet soaked with PBS (control), RANKL $\left(50 \mu \mathrm{g} \mathrm{ml}^{-1}\right)$ or with RANKL and recombinant Osteoactivin $\left(100 \mu \mathrm{g} \mathrm{m}^{-1}\right)$. A week later, calvarias were harvested and TRAP-stained to determine the presence of osteoclasts. The TRAP-stained area was quantified as described in the 'Materials and Methods' section (b). (c) Calvaria in a were analyzed by micro-computated tomography. Representative images are shown. Data presented in all graphs represent mean \pm s.e.m. ${ }^{* *} P<0.01$ compared with control.

but stimulates function through IL-1 $\beta$ and nitric oxide. ${ }^{57}$ Interestingly, fibronectin has been found to bind CD44 through its heparin-binding domain. ${ }^{58}$ It will be interesting to determine if $\mathrm{CD} 44$, Osteoactivin and fibronectin have any interaction or involvement in osteoclast differentiation and function.

Our data indicate that recombinant Osteoactivin inhibits osteoclast differentiation and recruitment in an inflammatory in vivo osteolysis model. Previous literature has shown several different anti-inflammatory components that suppress osteoclast formation in vivo. ${ }^{41-43}$ It is interesting to note that recombinant Osteoactivin has a negative response on osteoclast resorption in vivo, yet stimulated its function in vitro. There may be two separate explanations seen in this phenomenon. First, Osteoactivin may be inhibiting the recruitment and differentiation of osteoclasts directly resulting in a reduced number of osteoclasts and less resorption. Second, the results seen in vivo may be indirectly due to the effect of Osteoactivin on osteoblasts. Previous data from our lab have shown that addition of recombinant Osteoactivin in osteoblasts results in the reduction of RANKL and other inflammatory cytokines (data not shown). This is also supported by the fact that mutation in Osteoactivin results in an increase in RANKL expression. ${ }^{14}$ Therefore, it may be possible that Osteoactivin may be indirectly affecting the osteoclasts by reducing the production of RANKL and other inflammatory cytokines secreted by the osteoblasts. Future studies should focus on the dual roles of Osteoactivin in osteoblasts and osteoclasts using tissue-specific transgenic or knockout animals to define the role of Osteoactivin in these cell types.

Interestingly, deficiency of CD44 resulted in the abrogation of Osteoactivin-mediated inhibition of osteoclast differentiation and recruitment in vivo. Previous studies have shown that CD44 has a potential role as an anti-inflammatory component in different tissue types. ${ }^{59-61} \mathrm{~A}$ previous study has shown that CD44 can act as a critical inhibitor for joint destruction and 
inflammatory bone loss. ${ }^{23}$ Furthermore, the anti-inflammatory response through CD44 has been linked to TGF- $\beta .^{62-64}$ Interestingly, Osteoactivin has been shown to regulate TGF- $\beta$ levels as transgenic mice overexpressing Osteoactivin have increased levels of TGF- $\beta .{ }^{12}$ It will be interesting to determine if there is a link between Osteoactivin, CD44 and TGF- $\beta$ in reducing the inflammatory response in vivo.

Overall this study has shown that recombinant Osteoactivin inhibits osteoclast differentiation and that this effect seems to be influenced through its interaction with CD44. There is much attention being drawn to CD44 and Osteoactivin as repair factors. Previous literature has shown that CD44 and Osteoactivin are both upregulated in fracture calluses. ${ }^{33,65,66}$ Furthermore, previous data from our lab and others have shown that Osteoactivin stimulates bone formation and angiogenesis in vivo in a mouse and rat calvaria defect model. ${ }^{67,68}$ It will be interesting to determine if the interaction between CD44 and Osteoactivin can be translated in vivo. The use of fracture models in CD44 and Osteoactivin knockout models may help to identify the role of CD44 and Osteoactivin in bone repair.

\section{CONFLICT OF INTEREST}

The authors declare no conflict of interest.

1 Burge R, Dawson-Hughes B, Solomon DH, Wong JB, King A, Tosteson A. Incidence and economic burden of osteoporosis-related fractures in the United States, 2005-2025. J Bone Miner Res 2007; 22: 465-475.

2 Hammett-Stabler C. Bones and groans: the basics of osteoporosis and the use of bone markers. Lab Med 2007; 38: 273-278.

3 Raisz LG. Pathogenesis of osteoporosis: concepts, conflicts, and prospects. J Clin Invest 2005; 115: 3318-3325.

4 Safadi FF, Xu J, Smock SL, Rico MC, Owen TA, Popoff SN. Cloning and characterization of osteoactivin, a novel cDNA expressed in osteoblasts. J Cell Biochem 2001; 84: 12-26.

5 Abdelmagid SM, Barbe MF, Rico MC, Salihoglu S, Arango-Hisijara I, Selim AH, et al. Osteoactivin, an anabolic factor that regulates osteoblast differentiation and function. Exp Cell Res 2008; 314: 2334-2351.

6 Sheng MH, Wergedal JE, Mohan S, Lau KH. Osteoactivin is a novel osteoclastic protein and plays a key role in osteoclast differentiation and activity. FEBS Lett 2008; 582: 1451-1458.

7 Furochi H, Tamura S, Mameoka M, Yamada C, Ogawa T, Hirasaka K, et al. Osteoactivin fragments produced by ectodomain shedding induce MMP-3 expression via ERK pathway in mouse NIH-3T3 fibroblasts. FEBS Lett 2007; 581: 5743-5750.

8 Rose AA, Annis MG, Dong Z, Pepin F, Hallett M, Park M, et al. ADAM10 releases a soluble form of the GPNMB/Osteoactivin extracellular domain with angiogenic properties. PLOS ONE 2010; 5: e12093.

9 Yu B, Sondag GR, Malcuit C, Kim MH, Safadi FF. Macrophage-associated osteoactivin/GPNMB mediates mesenchymal stem cell survival, proliferation, and migration via a CD44-dependent mechanism. J Cell Biochem 2016; 117: 1511-1521.

10 Selim AA, Abdelmagid SM, Kanaan RA, Smock SL, Owen TA, Popoff SN, et al. Anti-osteoactivin antibody inhibits osteoblast differentiation and function in vitro. Crit Rev Eukaryot Gene Expr 2003; 13: 265-275.

11 Sondag GR, Salihoglu S, Lababidi SL, Crowder DC, Moussa FM, Abdelmagid SM, et al. Osteoactivin induces transdifferentiation of C2C12 myoblasts into osteoblasts. J Cell Physiol 2014; 229: 955-966.

12 Frara N, Abdelmagid SM, Sondag GR, Moussa FM, Yingling VR, Owen TA, et al. Transgenic expression of osteoactivin/gpnmb enhances bone formation in vivo and osteoprogenitor differentiation ex vivo. J Cell Physiol 2016; 231: 72-83.
13 Abdelmagid SM, Belcher JY, Moussa FM, Lababidi SL, Sondag GR, Novak KM, et al. Mutation in osteoactivin decreases bone formation in vivo and osteoblast differentiation in vitro. Am J Pathol 2014; 184: 697-713.

14 Abdelmagid SM, Sondag GR, Moussa FM, Belcher JY, Yu B, Stinnett H, et al. Mutation in osteoactivin promotes RANKL-mediated osteoclast differentiation and survival, but inhibits osteoclast function. J Biol Chem 2015; 290: 20128-20146.

15 Sheng MH, Wergedal JE, Mohan S, Amoui M, Baylink DJ, Lau KH. Targeted overexpression of osteoactivin in cells of osteoclastic lineage promotes osteoclastic resorption and bone loss in mice. PLOS ONE 2012; 7: e35280.

16 Miyazaki T, Miyauchi S, Anada T, Tawada A, Suzuki O. Chondroitin sulfate-E binds to both osteoactivin and integrin alphaVbeta3 and inhibits osteoclast differentiation. J Cell Biochem 2015; 116: 2247-2257.

17 Hughes DE, Salter DM, Simpson R. CD44 expression in human bone: a novel marker of osteocytic differentiation. J Bone Miner Res 1994; 9 39-44.

18 Jamal $\mathrm{HH}$, Aubin JE. CD44 expression in fetal rat bone: in vivo and in vitro analysis. Exp Cell Res 1996; 223: 467-477.

19 Nakamura H, Ozawa H. Immunolocalization of CD44 and the ERM family in bone cells of mouse tibiae. J Bone Miner Res 1996; 11: 1715-1722.

20 Reyes-Botella C, Montes MJ, Vallecillo-Capilla MF, Olivares EG, Ruiz C. Expression of molecules involved in antigen presentation and $T$ cell activation (HLA-DR, CD80, CD86, CD44 and CD54) by cultured human osteoblasts. J Periodontol 2000; 71: 614-617.

21 Noonan KJ, Stevens JW, Tammi R, Tammi M, Hernandez JA, Midura RJ. Spatial distribution of CD44 and hyaluronan in the proximal tibia of the growing rat. J Orthop Res 1996; 14: 573-581.

22 Nakamura H, Kenmotsu S, Sakai H, Ozawa H. Localization of CD44, the hyaluronate receptor, on the plasma membrane of osteocytes and osteoclasts in rat tibiae. Cell Tissue Res 1995; 280: 225-233.

23 Hayer S, Steiner G, Gortz B, Reiter E, Tohidast-Akrad M, Amling M, et al. CD44 is a determinant of inflammatory bone loss. J Exp Med 2005; 201: 903-914.

24 Cao JJ, Singleton PA, Majumdar S, Boudignon B, Burghardt A, Kurimoto P, et al. Hyaluronan increases RANKL expression in bone marrow stromal cells through CD44. J Bone Miner Res 2005; 20: 30-40.

25 de Vries TJ, Schoenmaker T, Beertsen W, van der Neut R, Everts V. Effect of CD44 deficiency on in vitro and in vivo osteoclast formation. J Cell Biochem 2005; 94: 954-966.

26 Suzuki K, Zhu B, Rittling SR, Denhardt DT, Goldberg HA, McCulloch CA, et al. Colocalization of intracellular osteopontin with CD44 is associated with migration, cell fusion, and resorption in osteoclasts. J Bone Miner Res 2002; 17: 1486-1497

27 Marroquin CE, Downey L, Guo H, Kuo PC. Osteopontin increases CD44 expression and cell adhesion in RAW 264.7 murine leukemia cells. Immunol Lett 2004; 95: 109-112.

28 Spessotto P, Rossi FM, Degan M, Di Francia R, Perris R, Colombatti A, et al. Hyaluronan-CD44 interaction hampers migration of osteoclast-like cells by down-regulating MMP-9. J Cell Biol 2002; 158: 1133-1144.

29 Sterling H, Saginario C, Vignery A. CD44 occupancy prevents macrophage multinucleation. J Cell Biol 1998; 143: 837-847.

30 Geldenhuys WJ, Novotny N, Malan SF, Van der Schyf CJ. 3D-QSAR and docking studies of pentacycloundecylamines at the sigma-1 (sigma1) receptor. Bioorg Med Chem Lett 2013; 23: 1707-1711.

31 Al-Baghdadi OB, Prater NI, Van der Schyf CJ, Geldenhuys WJ. Inhibition of monoamine oxidase by derivatives of piperine, an alkaloid from the pepper plant Piper nigrum, for possible use in Parkinson's disease. Bioorg Med Chem Lett 2012; 22: 7183-7188.

32 Chang EJ, Kim HJ, Ha J, Kim HJ, Ryu J, Park KH, et al. Hyaluronan inhibits osteoclast differentiation via Toll-like receptor 4. J Cell Sci 2007, 120: $166-176$.

33 Abdelmagid SM, Barbe MF, Hadjiargyrou M, Owen TA, Razmpour R, Rehman S, et al. Temporal and spatial expression of osteoactivin during fracture repair. J Cell Biochem 2010; 111: 295-309.

34 Boyle WJ, Simonet WS, Lacey DL. Osteoclast differentiation and activation. Nature 2003; 423: 337-342.

35 Tao J, Chen S, Lee B. Alteration of Notch signaling in skeletal development and disease. Ann N Y Acad Sci 2010; 1192: 257-268.

36 Ashley JW, Ahn J, Hankenson KD. Notch signaling promotes osteoclast maturation and resorptive activity. J Cell Biochem 2015; 116 : 2598-2609. 
37 Wada T, Nakashima T, Hiroshi N, Penninger JM. RANKL-RANK signaling in osteoclastogenesis and bone disease. Trends Mol Med 2006; 12: 17-25.

38 Blair HC, Athanasou NA. Recent advances in osteoclast biology and pathological bone resorption. Histol Histopathol 2004; 19: 189-199.

39 Teriete P, Banerji S, Noble M, Blundell CD, Wright AJ, Pickford AR, et al. Structure of the regulatory hyaluronan binding domain in the inflammatory leukocyte homing receptor CD44. Mol Cell 2004; 13: 483-496.

40 Lesley J, Hascall VC, Tammi M, Hyman R. Hyaluronan binding by cell surface CD44. J Biol Chem 2000; 275: 26967-26975.

41 Lee JH, Kim HN, Yang D, Jung K, Kim HM, Kim HH, et al. Trolox prevents osteoclastogenesis by suppressing RANKL expression and signaling. J Biol Chem 2009; 284: 13725-13734.

$42 \mathrm{Ha} \mathrm{H}$, Lee JH, Kim HN, Kim HM, Kwak HB, Lee S, et al. alpha-Lipoic acid inhibits inflammatory bone resorption by suppressing prostaglandin E2 synthesis. J Immunol 2006; 176: 111-117.

$43 \mathrm{Kim}$ HN, Lee JH, Jin WJ, Lee ZH. alpha-tocopheryl succinate inhibits osteoclast formation by suppressing receptor activator of nuclear factorkappaB Ligand (RANKL) expression and bone resorption. J Bone Metab 2012; 19: 111-120.

44 Yoon SH, Ryu J, Lee Y, Lee ZH, Kim HH. Adenylate cyclase and calmodulin-dependent kinase have opposite effects on osteoclastogenesis by regulating the PKA-NFATc1 pathway. J Bone Miner Res 2011; 26: 1217-1229.

45 Reunanen N, Li SP, Ahonen M, Foschi M, Han J, Kahari VM. Activation of p38 alpha MAPK enhances collagenase-1 (matrix metalloproteinase (MMP)-1) and stromelysin-1 (MMP-3) expression by mRNA stabilization. J Biol Chem 2002; 277: 32360-32368.

46 Greenblatt MB, Shim JH, Zou W, Sitara D, Schweitzer M, Hu D, et al. The p38 MAPK pathway is essential for skeletogenesis and bone homeostasis in mice. J Clin Invest 2010; 120: 2457-2473.

47 Tanaka S, Nakamura I, Inoue J, Oda H, Nakamura K. Signal transduction pathways regulating osteoclast differentiation and function. J Bone Miner Metab 2003; 21: 123-133.

48 Moon JB, Kim JH, Kim K, Youn BU, Ko A, Lee SY, et al. Akt induces osteoclast differentiation through regulating the GSK3beta/NFATc1 signaling cascade. J Immunol 2012; 188: 163-169.

49 Lee SE, Chung WJ, Kwak HB, Chung CH, Kwack KB, Lee ZH, et al. Tumor necrosis factor-alpha supports the survival of osteoclasts through the activation of Akt and ERK. J Biol Chem 2001; 276: 49343-49349.

50 Lee ZH, Lee SE, Kim CW, Lee SH, Kim SW, Kwack K, et al. IL-1alpha stimulation of osteoclast survival through the PI 3-kinase/Akt and ERK pathways. J Biochem 2002; 131: 161-166.

51 Vignery A. Osteoclasts and giant cells: macrophage-macrophage fusion mechanism. Int J Exp Pathol 2000; 81: 291-304.

52 Liu H, Cui J, Sun J, Du J, Feng W, Sun B, et al. Histochemical evidence of zoledronate inhibiting c-src expression and interfering with CD44/OPNmediated osteoclast adhesion in the tibiae of mice. J Mol Histol 2015; 46: 313-323.

53 Chellaiah MA, Hruska KA. The integrin alpha(v)beta(3) and CD44 regulate the actions of osteopontin on osteoclast motility. Calcif Tissue Int 2003; 72: 197-205.

54 Chellaiah MA, Biswas RS, Rittling SR, Denhardt DT, Hruska KA. Rho-dependent Rho kinase activation increases CD44 surface expression and bone resorption in osteoclasts. J Biol Chem 2003; 278: 29086-29097.

55 Chabadel A, Banon-Rodriguez I, Cluet D, Rudkin BB, Wehrle-Haller B, Genot $\mathrm{E}$, et al. CD44 and beta3 integrin organize two functionally distinct actin-based domains in osteoclasts. Mol Biol Cell 2007; 18: 4899-4910.

56 Tiedemann K, Boraschi-Diaz I, Rajakumar I, Kaur J, Roughley P, Reinhardt DP, et al. Fibrillin-1 directly regulates osteoclast formation and function by a dual mechanism. J Cell Sci 2013; 126: 4187-4194.

57 Gramoun A, Azizi N, Sodek J, Heersche JN, Nakchbandi I, Manolson MF. Fibronectin inhibits osteoclastogenesis while enhancing osteoclast activity via nitric oxide and interleukin-1beta-mediated signaling pathways. J Cell Biochem 2010; 111: 1020-1034.

58 Jalkanen S, Jalkanen M. Lymphocyte CD44 binds the $\mathrm{COOH}$-terminal heparin-binding domain of fibronectin. J Cell Biol 1992; 116: 817-825.

59 Johnson P, Ruffell B. CD44 and its role in inflammation and inflammatory diseases. Inflamm Allergy Drug Targets 2009; 8: 208-220.

60 Liang J, Jiang D, Griffith J, Yu S, Fan J, Zhao X, et al. CD44 is a negative regulator of acute pulmonary inflammation and lipopolysaccharide-TLR signaling in mouse macrophages. J Immunol 2007; 178: 2469-2475.

61 Kawana H, Karaki H, Higashi M, Miyazaki M, Hilberg F, Kitagawa M, et al. CD44 suppresses TLR-mediated inflammation. J Immunol 2008; 180: 4235-4245.

62 Teder P, Vandivier RW, Jiang D, Liang J, Cohn L, Pure E, et al. Resolution of lung inflammation by CD44. Science 2002; 296: 155-158.

63 Acharya PS, Majumdar S, Jacob M, Hayden J, Mrass P, Weninger W, et al. Fibroblast migration is mediated by CD44-dependent TGF beta activation. J Cell Sci 2008; 121: 1393-1402.

64 Yu Q, Stamenkovic I. Cell surface-localized matrix metalloproteinase-9 proteolytically activates TGF-beta and promotes tumor invasion and angiogenesis. Genes Dev 2000; 14: 163-176.

65 Yamazaki M, Nakajima F, Ogasawara A, Moriya H, Majeska RJ, Einhorn TA. Spatial and temporal distribution of CD44 and osteopontin in fracture callus. J Bone Joint Surg Br 1999; 81: 508-515.

66 Colburn NT, Zaal KJ, Wang F, Tuan RS. A role for gamma/delta T cells in a mouse model of fracture healing. Arthritis Rheum 2009; 60: 1694-1703.

67 Bateman JP, Safadi FF, Susin C, Wikesjo UM. Exploratory study on the effect of osteoactivin on bone formation in the rat critical-size calvarial defect model. J Periodontal Res 2012; 47: 243-247.

68 Hu X, Zhang P, Xu Z, Chen H, Xie X. GPNMB enhances bone regeneration by promoting angiogenesis and osteogenesis: potential role for tissue engineering bone. J Cell Biochem 2013; 114: 2729-2737.

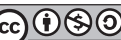

This work is licensed under a Creative Commons Attribution-NonCommercial-ShareAlike 4.0 International License. The images or other third party material in this article are included in the article's Creative Commons license, unless indicated otherwise in the credit line; if the material is not included under the Creative Commons license, users will need to obtain permission from the license holder to reproduce the material. To view a copy of this license, visit http:// creativecommons.org/licenses/by-nc-sa/4.0/

Supplementary Information accompanies the paper on Experimental \& Molecular Medicine website (http://www.nature.com/emm) 